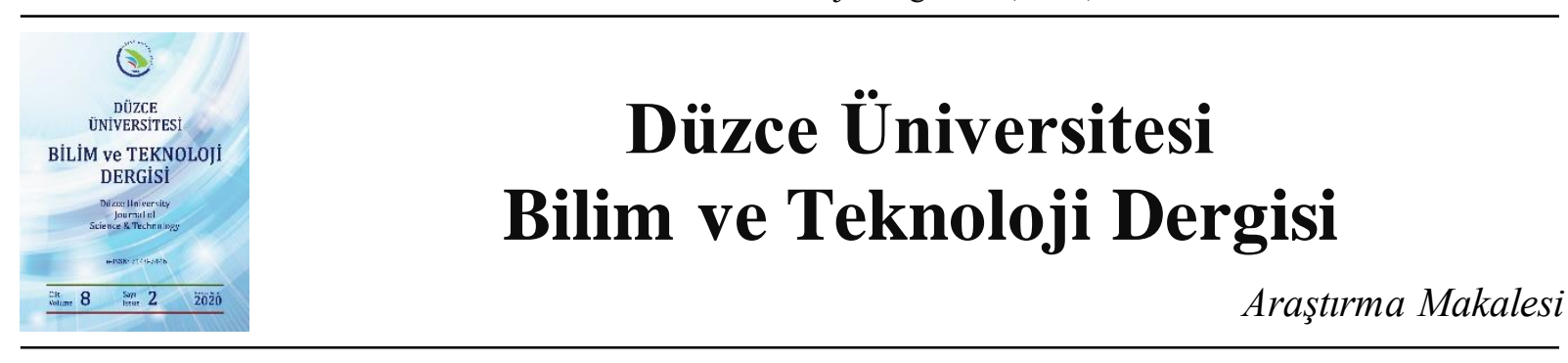

\title{
Pasif Milimetre Dalga Görüntüleme Sistemi için Radyometrik Alıcının Parabolik Yansıtıcı Antendeki Doğru Konumunun Belirlenmesi
}

\author{
iD Mehmet DUMAN ${ }^{a}{ }^{*}$, (D) Alp Oral SALMAN b \\ ${ }^{a}$ Elektrik Elektronik Müh. Bölümü, Teknoloji Fakültesi, Düzce Üniversitesi, Düzce, TÜRKIYYE \\ ${ }^{b}$ Elektronikve Haberleşme Müh. Bölümü, Mühendislik Fakültesi, Kocaeli Üniversitesi, Kocaeli, TÜRKIYYE \\ * Sorumlu yazarin e-posta adresi: mehmetduman@duzce.edu.tr
}

DOI: $10.29130 /$ dubited.643743

\begin{abstract}
ÖZET
Savunma ve navigasyon gibi a lanlarda, optik ve kızılötesi görüntüleme sistemlerinin sağlıklı çalışamadığı sis, yağmur ve toz gibi görüşü engelleyen çeşitli hava olaylarında, ortamı görüntülemek için, tamamlayıcı sensör olarak Pasif MiliMetre Dalga Görüntüleme Sistemi (PMMDGS) kullanılabilir. Sistem; radyometrik alıcının parabolik yansıtıcı anteninin odak nokta sında bulunduğu ve bu antenin iki eksende (azimut ve ka lkış) ça lışan bir konumlandırıcı tarafından taranmasıyla görüntülemenin yapıldı ğı bir teknolojiye sahiptir. Bir PMMDGS'de görüntülenen ortamdan gelen sinya lin oldukça zayıf olmasından dolayı hem iyi bir kuvvetlendirmenin yapılması hem de alıcıdan optimal sinyalin elde edilebilmesi için radyometrik alıcının parabolik yansıtıcı antenin odak noktasına doğru konumunda yerleştirilmesi büyük bir önem arz etmekte dir. Bu çalışmada; PMMDGS'nin anteni, gökyüzü gibi sabit bir görüntüleme noktasına yönlendirilmiş iken radyometrik alıcı, parabolik yansıtıcı antenden belli mesafelerde kaydırılmış ve optimal sinyalin elde edildiği konum bulunmuştur. Sonuçlar, algılamanın radyometrik alıcıya bağlı olan horn antenin açıklık ucundan değil, bağlantı kısmına yakın bir noktadan olduğunu göstermiştir.
\end{abstract}

Anahtar Kelimeler: Odak noktasl, Parabolik yansitıcı anten, Pasif milimetre dalga görüntüleme sistemi, Radyometrikalıcl

\section{Determining the Correct Position of the Radiometric Receiver on the Parabolic Reflector Antenna for the Passive Millimeter Wave Imaging System}

\begin{abstract}
In areas such as defense and na vigation; a variety of weather events that prevent vision, such as fog, rain, and dust, where optical and infrared imaging systems cannot work properly, the Passive MilliMeter Wave Imaging System (PMMWIS) can be used a a complementary sensor to display the environment. Th is system where the radiometric receiver is located at the focalpoint of the parabolic reflector antenna and the antenna is scanned by a positioner operating on the two axis (azimuth and elevation) is very technological. Because the signal from the scenario displayed in a PMMWIS is rather weak; in order to achieve a good amplification and to obtain the optimal signal from the receiver, it is of great importance that the ra dio metric receiver should be positioned in the correct position of the parabolic reflector antenna. In this study; when the PMMWIS antenna was looking at a fixed viewing point, such as sky, the radiometric receiver was shifted at certain distances from the pa rabolic reflector antenna and the
\end{abstract}


position where the optimal signal was obtained. The results showed that the detection was from a point close to the connecting part, not the aperture end of the horn antenna connected to the radiometric receiver.

Keywords: Focal point, Parabolic reflector antenna, Passive millimeter wave imaging system, Radiometric receiver

\section{GíRis}

Pasif MiliMetre Dalga Görüntüleme Sistemi (PMMDGS), güvenlik alanında kullanılabilen ve son yıllarda araştırmaların yoğunlaştığı yeni bir teknolojidir [1 -4]. Bu sistem, uzakta bulunan cisimlerin görüşü engelleyen sisli, tozlu ve dumanlı ortamşartlarında, optik vekızılötesi görüntüleme sistemlerinin yetersiz kaldığı durumlarda yardımcı bir görüntüleme sistemi olarak çalışabilmektedir. Bu işlemi gerçekleştirebilmesinin temel nedeni ise sis ve yağmur zayıflatmalarının yüksek frekanslara göre radyo frekanslarında oldukça düşmesidir [5]. Ancak; bu frekanslarda kara cisim [6] fiziksel temeline dayanan pasif algılama $[7,8]$ sinyalinin de çok düşmesinden dolayı bu sinyalin yeterince kuvvetlendirilmesi de elzem hale gelmektedir $[9,10]$. Savunma, navigasyon gibi alanlarda kullanılabilen bu sistem; tek bir alıcının iki eksende tarama yaptırılarak görüntü elde edilmesiyle çalışmaktadır.

Bir PMMDGS'de parabolik yansitıcı antene [11] gelen sinyaller, antenin odak noktasında bulunan bir radyometrik alıc $[12,13]$ tarafından alınır. Alıcıdan elde edilen sinyalin maksimum seviyede olması için, alıcının parabolik yansıtıcı antenin odak noktasına doğru konumunda yerleştirilmesi gerekir. $\mathrm{Bu}$ çalışmada, doğru konumun bulunmasına yönelik ölçümler ve ölçümlerin değerlendirilmesi anlatılacaktır. Bu sayede de radyometrik alıcının doğru konumunun belirlenip, sistemin daha verimli şekilde çalışması sağlanmış olacaktır.

$\mathrm{Bu}$ makalenin girişten sonra yer alan ikinci bölümünde, ölçümlerin gerçekleştirildiği PMMDGS kullanılan malzemeler ile birlikte detaylı olarak aktarılmış; üçüncü bölümünde alıcının doğru konumunun bulunması için gerçekleştirilen ölçümler grafiklerle beraber izah edilmiş, yöntem ve bulgular aktarılmış, dördüncü bölümünde ise elde edilen sonuçlar tartışılmıştır.

\section{MALZEMELER VE PASIF MILIMETRE DALGA GÖRÜNTÜLEME SİSTEMİ}

Radyometrik alıcının parabolik yansıtıcı antenin odak noktasına doğru konumunda yerleştirilebilmesi için yapılan ölçümler Şekil 1.'deki PMMDGS yardımıyla gerçekleştirilmiştir. Sistemde, üç ayağa bağl olan ve üzerinde bir radyometrik alıcı bulunan parabolik yansitıcı antenin iki eksenli konumlandırcı vasıtasıyla tarama yapılarak bilgisayar ekranında görüntü elde edilmektedir. Elde edilen sinyalin ekrana aktarılması ve tarayıcının kontrolü elektronik kontrol ünitesi ile gerçekleştirilir. 


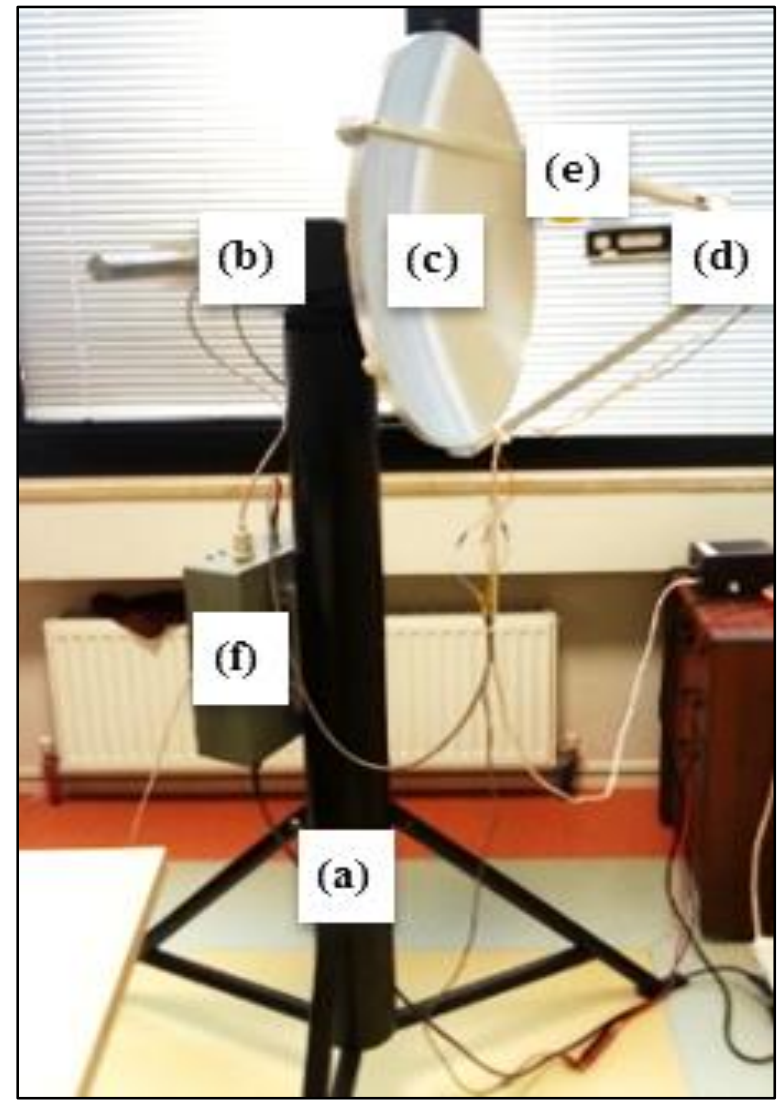

Şekil 1. PMMDGS, tüm sistem (a) Ü̧̧ ayak(b) 2 eksenli konumlandırıcı (c) Parabolikyansıtıcı anten (d) Antenin üzerine takıldiğ lalıcı tutucusu (e) Radyometrik alıcı (f) Kontrol ünitesi

Sistemin parabolik yansıtıcı anteni başka bir çalışmada tasarlanmış ve gerçekleştirilmiştir. Tasarlanmış olan bu parabolik yansıtıcı antene, $96 \mathrm{GHz}$ frekansında çalışan radyometrik alıcı ince metalik tutucular sayesinde tutturulmuştur. Ucunda bir horn anten bulunan radyometrik alıc1 Şekil 2. (a)'da ve üzerine radyometrik alıcı monte edilmiş parabolik yansıtıcı anten Şekil 2. (b)'de verilmiştir. Sistemin parabolik yansıtıcı anteninin çapı 0.50 metre, odak uzaklığı 0.25 metredir $(f / D=0.5)$.

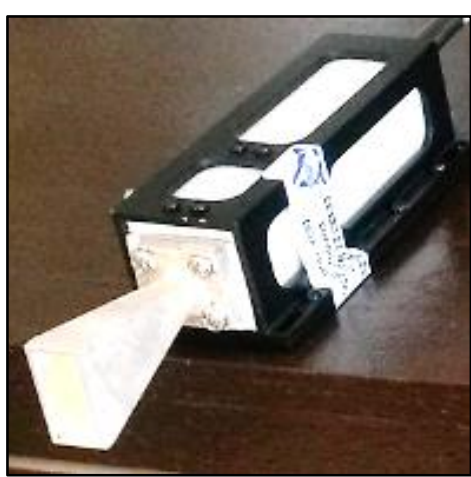

(a)

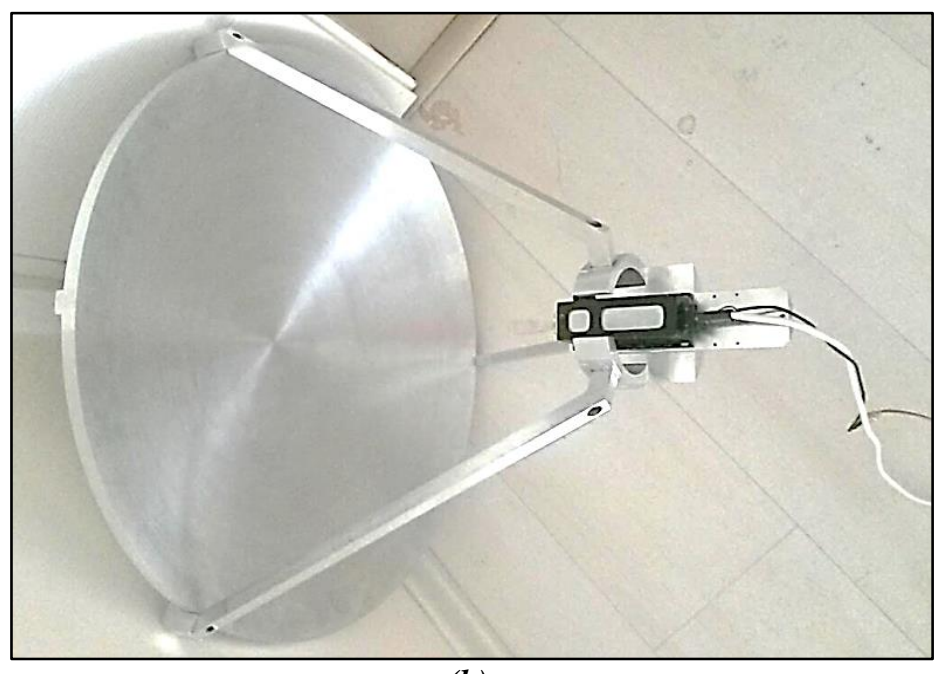

(b)

Şekil 2. (a) $96 \mathrm{GHz}$ frekansında çalışan radyometrik alıcı (b) Üzerine radyometrik alıcı monte edilmiş parabolik yansiticianten 


\section{YÖNTEM, ÖLCÜMLER VE BULGULAR}

Radyometrik alıcının parabolik yansıtıcı antenin odak noktasında doğru konumuna yerleştirilmesi amacıyla iki adet ölçüm yapılmıştır. Ölçümler esnasında çekilmişolan bir fotoğraf Şekil 3.'te verilmiştir. Ölçümler, anten tarama sisteminin el verdiği yataydan en büyük kalkış açısı olan $\theta=75^{\circ}$ olacak şekilde konumlandırılmıştır. Radyometrik alıcının gerilim çıkışı DC voltmetreye bağlanmış ve alıcının kendi kıză̆ üzerinde kaydırılırken, her konum için gerilim değerleri not edilmiştir.

Ölçümlerdeki mesafe, radyometrik alıcının horn anteninin ucundan, çanağın en çukur noktasına olan mesafedir. İlk ölçümde, çanağın en çukur noktasından $18.9 \mathrm{~cm}$ ile $31.5 \mathrm{~cm}$ mesafeleri arasında alıcının çıkış voltajı ölçümleri alınmıştır (Şekil 4. (a)). Bu ölçümde, maksimum voltajın $19.5 \mathrm{~cm}$ 'de1200 mV olarak gözlemlenmesine rağmen, tepe noktası tam olarak gözlemlenemediğinden; çanağa biraz daha yaklaşılarak, alıcıyı kaydırmaya $14 \mathrm{~cm}$ 'den başlanmış ve $30 \mathrm{~cm}$ 'e kadar ikinci bir ölçüm gerçekleştirilmiştir (Şekil 4. (b)).

Ölçüm noktalarına uygulanan Lorentz eğri uygunlaştırması sonucunda maksimum voltajın $19.1 \mathrm{~cm}$ 'de $1735 \mathrm{mV}$ olduğu bulunmuştur. Her iki ölçümden elde edilen sonuçlardan hareketle, bu mesafe değeri kolay kullanım açısından yuvarlanarak $20 \mathrm{~cm}$ olarak belirlenmiştir.

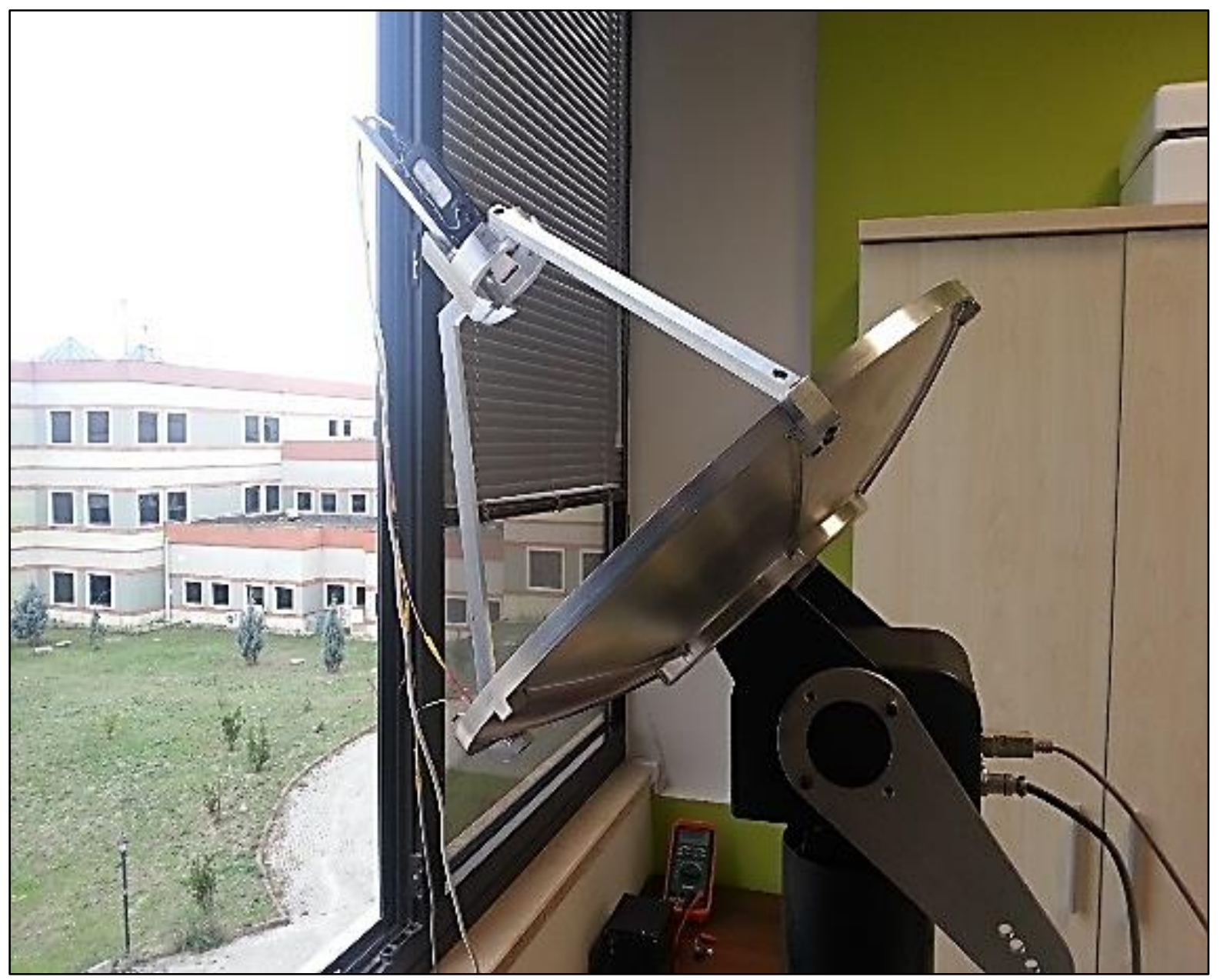

Şekil 3. Ölçümler esnasından birfotoğraf 


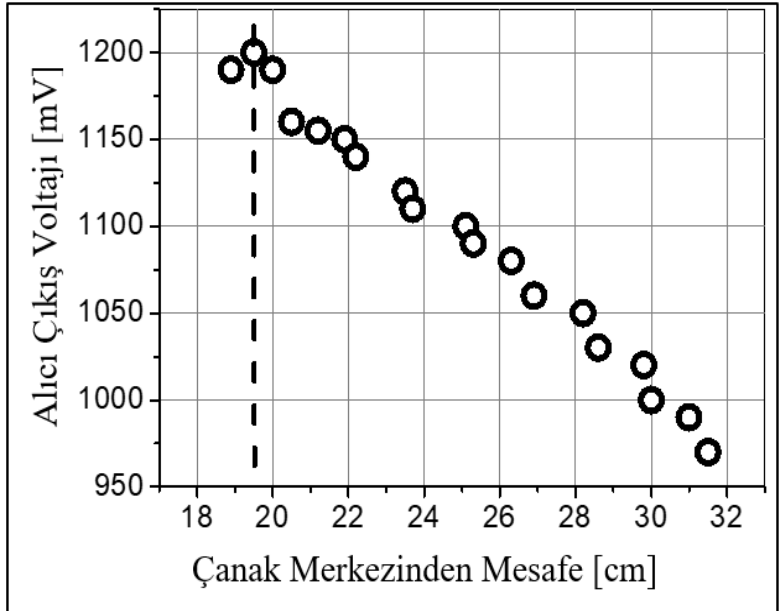

(a)

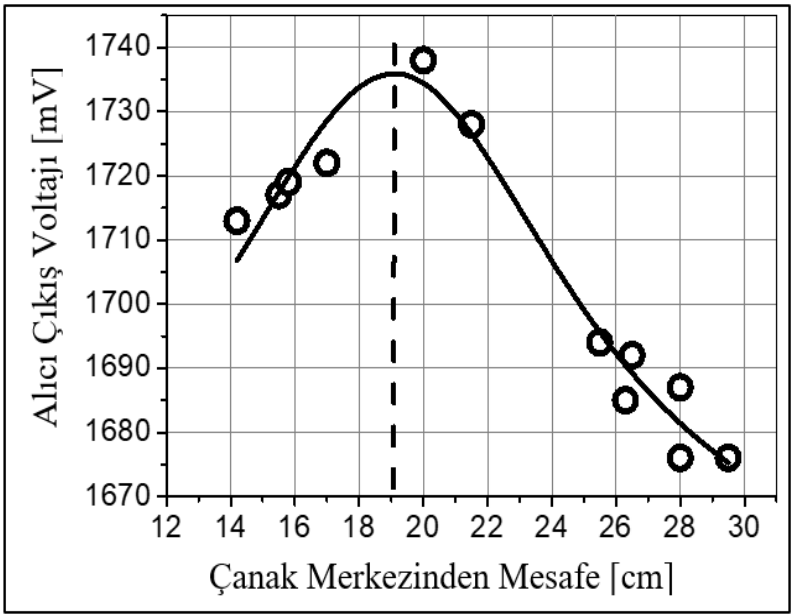

(b)

Şekil 4. Alıcının mesafesine göre çıkış voltajı grafikleri (a) 1. Ölçüm (b) 2. Ölçüm

\section{SONUCLAR}

Radyometrik alıcıya bağlı olan horn antenin uzunluğu yaklaşık $4.5 \mathrm{~cm}$ 'dir. Ölçümler sonucunda alıcı çıkışında maksimum voltajı elde edebilmek için parabolik yansıtıcı antenin odak noktasına horn antenin açıklık ağzının değil; horn antenin bağlantı ucunun, odak noktasına denk getirilmesi gerektiği görülmektedir. Buradan elde edilen sonuç; horn antenlerde, antenin üzerine gelen elektromanyetik dalganın açıklık ağzında değil; bağlantı ucunda algılandığıdır. Bu çalışma sayesinde; radyometrik alıcının, parabolik yansıtıcı antenin odak noktasındaki doğru konumu belirlenmiş̦tir. Buradan elde edilen diğer sonuç ise horn anten çıkartılarak sadece radyometrik alıcının dalga kılavuzu ağzı kullanılarak bir ölçüm yapılmak istenmesi durumunda; tam bu açıklık ağzının parabolik yansıtıcı antenin odaknoktasına denk getirilmesi gerektiğidir.

TEȘEKKÜR: $\quad \mathrm{Bu}$ çalışma Kocaeli Üniversitesi Bilimsel Araştırma Projeleri tarafından desteklenmiştir (Proje no: 2013/18). Yazarlardan Mehmet DUMAN TÜBİTAKBİDEB'e doktora burs desteği için teşekkür eder.

\section{KAYNAKLAR}

[1] L. Yujiri, M. Shoucri, P. Moffa, "Passive millimeter-wave imaging," Microwave Magazine IEEE, c. 4, s. 3, ss. 39-50, 2003.

[2] R. Appleby, R. N. Anderton, "Millimeter-Wave and Submillimeter-Wave Imaging for Security and Surveillance," Proceedings of the IEEE, c. 95, s. 8, ss. 1683-1690, 2007.

[3] A. Denisov ve diğ., "Some experiments concerning resolution of 32 sensors passive $8 \mathrm{~mm}$ wave imaging system," 20th. International Symposium on Space Terahertz Technology (ISSTT), Virginia, USA, 2009, ss. 227-229.

[4] A. Vertiy, ve diğ., "Short and Long Range Passive Imaging in Millimeter-Wave-Band," IEEE General Assembly and Scientific Symposium (XXXth URSI), İstanbul, Türkiye, 2011, ss. 1-4.

[5] L. Duixian, G. Brian, P. Ulrich, G. Janusz, Advanced Millimeter-Wave Technologies, Antennas, Packaging and Circuits, Wiley Publishing, 2009. 
[6] M. Duman, A.O. Salman, "Theoretical Investigation of Blackbody Radiation for the Passive Millimeter wave Imaging System," 2. International Symposium on Multidisciplinary Studies and Innovative Technologies (ISMSIT), Ankara, Türkiye, 2018, ss. 1-4.

[7] K. P. Kumar, Ground-Based Microwave Radiometry and Remote Sensing, Methods and Applications, CRC Press, 2014.

[8] C. Elachi, Introduction to the Physics and Techniques of Remote Sensing, Wiley Series in Remote Sensing, 1987.

[9] G. Mehdi, ve diğ., “A Modular Ka-Band Front-End Receiver for Passive Imaging System,” 16th International Multi Topic Conference(INMIC), Lahore, Pakistan, 2013.

[10] K. Mizuno, ve diğ., "New Appications of Millimeter-Wave Incoherent Imaging," IEEE MTT-S International Microwave Symposium Digest, Long Beach, CA, USA, 2005.

[11] C. A. Balanis, Anten Teorisi Analiz ve Tasarım, 3. bask1, Nobel Yayınları, 2013.

[12] N. Skou, D. Le Vine, Microwave Radiometer Systems, Design and Analysis, 2. Bask1, Artech House, 2006.

[13] F. T. Ulaby, R. K. Moore, A. K. Fung, Microwave Remote Sensing, Active and Passive, Volume 1, Microwave Remote Sensing Fundamentals and Radiometry, Artech House Inc., 1981. 